\section{Von der öffentlichen Finanzkontrolle lernen}

\author{
Rainer W. Wieschollek
}

Für die Durchführung eines Betriebsvergleichs können Organisationen der Sozialwirtschaft aus der überörtlichen Prüfung kommunaler Körperschaften lernen. Dazu sollte jedoch der ausschließlich quantitative Kennzahlenvergleich um die Analyse spezifischer Sachverhalte sozialer Leistungserbringer und um Vorschläge zur Behebung von Schwachstellen erweitert werden.

Die überörtliche Prüfung ist im Gegensatz zur örtlichen Eigenprüfung eine Prüfung durch eine externe Stelle. Bei der Organisation der überörtlichen Prüfung kommunaler Körperschaften haben sich bundesweit verschiedene Organisationsmodelle gebildet. Sie wird in den 13

Flächenstaaten beispielsweise durch eigenständige Prüfungseinrichtungen oder durch eine dem Innenressort zugeordnete Prüfungsbehörde durchgeführt. (1) In Hessen wird die Prüfung an Wirtschaftsprüfer oder andere geeignete Dritte durch den Präsidenten des Rechnungshofs vergeben. (2)

Wesentlicher Inhalt ist die mittelfristige Betrachtung und Bewertung der Verwaltungstätigkeit einer Kommune mit dem Schwerpunkt auf die Haushalts- und Wirtschaftsführung. Darüber hinaus hat die überörtliche Prüfung den Vorteil der vergleichenden Betrachtung. Die Prüfungen werden also innerhalb eines definierten Prüfungszeitraums in verschiedenen kommunalen Körperschaften durchgeführt und für einen interkommunalen Vergleich genutzt.

In Hessen wurden seit den Jahren 1993/94 bislang mehr als 80 vergleichende überörtliche Prüfungen kom- munaler Körperschaften auf der Grundlage des Gesetzes zur Regelung der überörtlichen Prüfung kommunaler Körperschaften (ÜPKKG) durchgeführt. Im Durchschnitt waren je Prüfung etwa 15 kommunale

Körperschaften beteiligt. (3) Neben der vergleichenden Betrachtung ist bei diesen Prüfungen kennzeichnend, dass wesentliche Daten und Feststellungen den beteiligten Körperschaften zuordenbar sind, also nicht anonymisiert werden. Damit gewinnt die Prüfung ein breites Spektrum an Erkenntnissen und Erfahrungen aus der kommunalen Praxis für die staatliche Finanzkontrolle aber auch für die beteiligten Körperschaften.

Der Präsident des Hessischen Rechnungshofs sieht beispielsweise folgende Vorteile in vergleichenden Untersuchungen (4):

- vergleichende Betrachtung

- Erstellen steuerungsrelevanter Benchmarks

- Einschätzung zur Lage der Kommunen

- spezielles Know-how durch externe Vergabe

Die Merkmale und Vorteile der überörtlichen Prüfung können gut für Betriebsvergleiche in der Sozialwirtschaft genutzt werden, wie nachfolgend dargelegt wird.

\section{Zielsetzung}

Abgeleitet aus den überörtlichen Prüfungen richtet sich das Ziel des internen und externen Betriebsvergleichs danach, ob die zu untersuchenden Einrichtungen jeweils für sich betrachtet (interner Vergleich) sowie auf vergleichenden Grundlagen

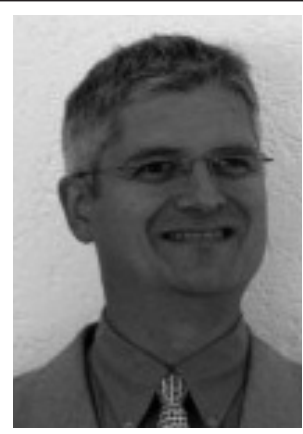

Der Diplom-Finanzwirt und Diplom-Verwaltungswissenschaftler Rainer Wieschol-

lek ist seit mehr als 15 Jahre in der Effizienzberatung von Organisationen der Sozialwirtschaft und der öffentlichen Verwaltung tätig. Schwerpunktmäßig ist er in Projekten zu Wirtschaftlichkeits-, Organisations- und Restrukturierungsfragen sowie der externen Finanzkontrolle tätig. Von 1990 bis 2004 war er als Berater in einem Berliner Beratungsinstitut und anschließend in einer der größten deutschen und internationalen Wirtschaftsprüfungsgesellschaft in Frankfurt am Main und München beschäftigt; zuletzt als Prokurist und Leiter der Verbändeberatung. Seit 2004 ist er als freiberuflicher Berater und externer Sachverständiger für Non-Profit-Organisationen und kommunalen Körperschaften in eigener Verantwort tätig.

E-Mailwieschollek@emcons.de

(externer Vergleich) ihre Aufgaben unter Berücksichtigung selbstgesetzter und gesetzlicher Standards

- sachgerecht (effektiv) und

- wirtschaftlich (effizient) erfüllen.

Des Weiteren können Fragen der Rechtmäßigkeit des Handelns und Aussagen über die Ordnungsmäßigkeit der Geschäftsführung (5) einer Einrichtung eine Rolle spielen.

Vor dem Hintergrund der Wirtschaftlichkeitsbetrachtung von Organisationen ist grundsätzlich zu berücksichtigen, dass es eine absolute Wirtschaftlichkeit nicht gibt - vielmehr werden Wirtschaftlichkeitskennzahlen erst im Vergleich aussagekräftig. Um zu sinnvollen Aussagen 
zu kommen, sollte der interne und externe Betriebsvergleich daher mindestens fünf oder mehr vergleichbare Einrichtungen einbeziehen. Die Offenlegung von Einsparungspotenzialen sowie die Verbesserung der Leistungsfähigkeit und Wirtschaftlichkeit stehen bei diesen Untersuchungen im Mittelpunkt.

Kennzeichnend für den hier skizzierten Betriebsvergleich ist aber, dass er sich nicht nur mit dem Vergleich von Kennzahlen, deren Kommentierung und der Diagnose von Verbesserungspotenzialen beschäftigt, sondern auch die Beratung und Empfehlungen für deren Nutzung und Weiterentwicklung für die beteiligten Organisationen zum Ziel hat. (6)

\section{Projektablauf}

Bei der Auswahl der zu untersuchenden Einrichtungen ist darauf zu achten, dass es sich auch um vergleichbare Einrichtungsarten oder Organisationen (z. B. Werkstätten für behinderte Menschen, ambulante Dienste oder Pflegeheime), vergleichbare Betriebsgrößen und regionale Bedingungen handelt. (7) Gegebenenfalls kann eine spätere Bildung von Clustern sinnvoll sein.

Die Durchführung des internen und externen Betriebsvergleichs erfolgt teilweise vor Ort unter Einbeziehung der Beteiligten in den Einrichtungen. Es hat sich eine

Vorgehensweise bewährt, die sich auf die im Folgenden beschriebenen drei Phasen konzentriert.

\section{Phase 1: Bestandsaufnahme und Analyse in einer Einrichtung (Piloteinrichtung)}

Die erste Untersuchung dient dazu, die Untersuchungsergebnisse zur Piloteinrichtung zu erarbeiten, festzustellen und für sich betrachtet zu bewerten. Des Weiteren kann in dieser Phase das Untersuchungskonzept (Methoden und Instrumente) in seiner Anwendbarkeit bestätigt und für den Vergleich weiterentwickelt werden. Mit Bestandsaufnahme und Analyse werden in der Piloteinrichtung die relevanten Merkmale und
Bestimmungsfaktoren der Einrichtung analysiert.

Vier Themenbereiche stehen im Fokus der Analyse: Leistungsdaten, Personaldaten, Finanzdaten, Organisationsregeln. Daraus ergeben sich mindestens die folgenden Untersuchungsfelder:

- grundsätzliche Programm- und Aufgabenstrukturen, einschließlich Standorte der Aufgabenerledigung

- Organisations- und Aufgabenstrukturen innerhalb einzelner Aufgaben- und Funktionsbereiche einschließlich wesentliche Geschäftsprozesse

- Führungsstrukturen

- Struktur der Leistungen und Auslastungen

- Personalwirtschaft

- finanzielle und wirtschaftliche Strukturen in Verbindung mit Sachmitteleinsatz, Beschaffung und Investitionen in Liegenschaften

- Schnittstellen zu anderen Einrichtungen

- Einsatz betriebswirtschaftlicher Instrumente (z. B. Controlling, Berichtswesen)

- Qualitätsmanagement

In Bestandsaufnahme und Analyse sollten bereits vorhandene Unterlagen und Dokumente einfließen (z. B. Berichte, Daten aus dem Rechnungswesen, Personal- und Leistungsstatistiken, Revisionsberichte, Kennzahlen aus bereits durchgeführten quantitativen Betriebsvergleichen), um den Erhebungsaufwand in einem verträglichen Maß zu halten. VorOrt-Begehungen und strukturierte Interviews mit einzelnen Personen der untersuchten Einrichtung runden die Bestandsaufnahme ab. Gegebenenfalls verfügt die Einrichtung bereits über ein differenziertes Controllingsystem und Berichtswesen, aus dem die erforderlichen Daten gewonnen werden können.

Zur kritischen Beurteilung der Sachgerechtheit (Effektivität) und Wirtschaftlichkeit (Effizienz) der untersuchten Bereiche wird ergänzend ein Kennzahlensystem zur Einzelbetrachtung der Piloteinrichtung entwickelt und ermittelt. Dieses Kennzahlensystem wird im Verlauf der Untersuchung auch und vor allem zum Quervergleich aller zu untersuchenden Einrichtungen (vgl. Phase 2: externer Vergleich) herangezogen. Der Quervergleich zwischen den Einrichtungen auf Basis des Kennzahlen- systems bezieht sich in der Regel auf das letzte vorliegende Geschäftsjahr in Form einer Zeitpunktbetrachtung. Zahlreiche auf ein Kalenderjahr bezogene Kennzahlen können in diesem Zusammenhang von Bedeutung sein (vgl. Tabelle). Ergänzend erfolgt ein Zeitvergleich über einen drei- bis vierjährigen Zeitraum (Zeitraumbetrachtung), um die Entwicklung einzelner Kennzahlen verfolgen und nachvollziehen zu können.

\section{Phase 2: Untersuchung weiterer Einrichtungen, externer Vergleich}

Nach Vorlage der Ergebnisse und Erkenntnisse aus der Piloteinrichtung werden die Untersuchungsfelder der übrigen Einrichtungen untersucht. Der Ablauf dieser Phase entspricht dabei im Grundsatz den Arbeitsschritten der Pilotuntersuchung. Art und Umfang der Erhebung werden gegebenenfalls angepasst; dies kann bei Bedarf Nacherhebungen zu einzelnen Aspekten in der Piloteinrichtung zur Folge haben. Entscheidend ist dabei, dass die Untersuchungsergebnisse (insbesondere Kennzahlen) aus allen untersuchten Einrichtungen miteinander verglichen werden. Ein-

\section{Vorteile}

Gegenüber dem System der überörtlichen Prüfung oder der in $\S 79$ SGB XI definierten Wirtschaftlichkeitsprüfung* hebt der hier beschriebene interne und externe Betriebsvergleich stärker ab auf

- Kundenorientierung

- Freiwilligkeit der Teilnahme an der Untersuchung

- aktive Einbindung der beteiligten Personen aus den Einrichtungen

- Kommentierung der Ergebnisse

- Entwicklung von Verbesserungsvorschlägen

- Rat geben statt Prüfen Rainer Wieschollek

* Z. B. Wirtschaftlichkeitsprüfung nach § 79 SGB XI; vgl. dazu auch Ausführungen von Burk, Rainer: Wie wirtschaftlich sind Alteneinrichtungen? In: neue caritas 10/2007. 
Kennzahlenbeispiele nach Bereichen

\begin{tabular}{|c|c|}
\hline \multicolumn{2}{|c|}{ Kennzahlenbeispiele nach Bereichen } \\
\hline Kapazitäts- und Leistungskennzahlen & Kennzahlen zur Gewinn- und Verliustrechnung \\
\hline $\begin{array}{ll}\text { - } & \text { Leistungsstruktur } \\
\text { - } & \text { (administrative und operative Bereiche) } \\
\text { - } & \text { Aundenstruktur } \\
\text { - } & \text { Betreuungsschlüssel (Ist/Soll) } \\
\text { - } & \text { Abrechnungen Plätze je Vollkraft } \\
\text { - } & \ldots\end{array}$ & 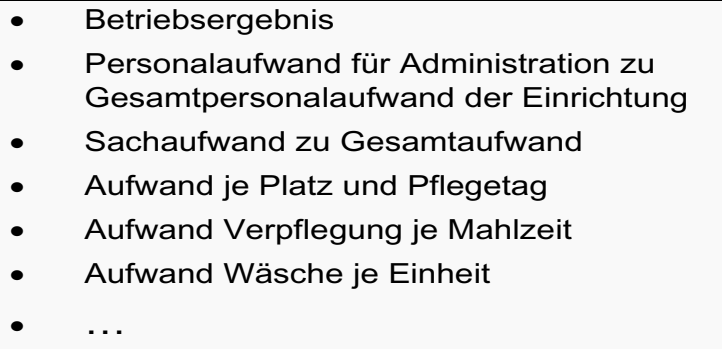 \\
\hline Personalkennzahlen & Bilanzkennzahlen \\
\hline $\begin{array}{ll}\text { - } & \text { Personalstruktur nach Bereichen } \\
\text { - } & \text { Fachkräfteanteil } \\
\text { - } & \text { Personalaufwand je Vollkraft } \\
\text { - } & \text { Besetzte Stellen zu finanzierten Stellen } \\
\text { - } & \text { Durchschnittliche Brutto- und Nettojahresarbeits- } \\
\text { - } & \text { Deit je Vollkraft } \\
\text { - } & \text { Durchschnittliche Ausfallzeiten }\end{array}$ & $\begin{array}{ll}\text { - } & \text { Anteile für Fremd- und Eigenfinanzierung } \\
\text { - } & \text { Verschuldungsgrad } \\
\text { - } & \text { Liquidität } \\
\text { - } & \text { Investitionsquote } \\
\text { - } & \ldots\end{array}$ \\
\hline
\end{tabular}

zelwerte und Durchschnittsgrößen, über- und unterdurchschnittliche $\mathrm{Ab}$ weichungen werden ermittelt und kommentiert.

\section{Phase 3: Rahmenkonzept mit Vor- schlägen zur Weiterentwicklung der untersuchten Einrichtungen}

Im dritten Projektschritt erfolgt die Entwicklung und Skizzierung von umsetzungsfähigen Vorschlägen zur Weiterentwicklung der untersuchten Organisationseinheiten in einem schriftlichen Bericht (Rahmenkonzept). Inhaltliche Ausführungen im Rahmenkonzept sind:

- Beschreibung der durchgeführten Untersuchungsarbeiten

- Hinweise auf angewandte Beurteilungsmaßstäbe

- Darstellung der vorgefundenen Sachverhalten, vor allem ihrer wirtschaftlichen Relevanz, einschließlich der nach (ggf. anonymisierten) Einrichtungen geordneten Kennzahlen

- Identifizierung veränderungsbedürftiger Sachverhalte, Strukturen, Kapazitäten, Geschäftsprozesse, sonstiger Leistungsprozesse und Kosten

- Aufzeigen sonstiger kosten- und erlösrelevanter Auffälligkeiten, einschließlich Tipps zu rasch umsetzbaren Sofortmaßnahmen
- Vorschläge und Priorisierung für Entwicklungsmaßnahmen

- Abschätzung des wirtschaftlichen Potenzials der vorgenannten Ansatzpunkte durch die Weiterentwicklung der Organisationen

\section{Fazit}

Die Sozialwirtschaft kann einzelne Bestandteile der Prüfungsmethoden und Prüfungsinhalte aus der überörtlichen Prüfung in einer auf den Untersuchungsgegenstand zugeschnittenen Form sinnvoll nutzen. Dann wird der interne und externe Betriebsvergleich die Transparenz der einzelnen Einrichtungen sowohl auf der Aufwands- und Erlösseite, als auch auf der Leistungsseite und durch die Kombination dieser Aspekte miteinander deutlich erhöhen. Insbesondere die daraus abgeleitete Entwicklung eines fortschreibungsfähigen Kennzahlensystems versetzt die beteiligten Einrichtung und deren Träger in die Lage, ihr eigenes Wirtschaftlichkeitsverhalten dauerhaft und nachhaltig zu steuern, um sachgerecht und wirtschaft Handeln zu können.

\section{Anmerkungen}

(1) Vgl. Kommunalprüfung; Veröffentlichung des Landesrechnungshofs Schleswig-Holstein, S. 2 ff., Stand: März 2005.
(2) Vgl. § 5 Abs. 1 Satz 4 ÜPKKG (Gesetz zur Regelung der überörtlichen Prüfung kommunaler Körperschaften vom 22.Dezember 1993)

(3) Vgl. Ersten bis Sechzehnten »Zusammenfassenden Bericht über die Feststellungen von allgemeiner Bedeutung bei der überörtlichen kommunalen Prüfung von 1994/95 bis 2006 « des Hessischen Rechnungshofs. In: Website des Hessischen Rechnungshofs, Veröffentlichungen (www.rechnungshof-hessen.de).

(4) Vgl. Eibelshäuser, Manfred: Erfahrungen mit vergleichenden Prüfungen kommunaler Körperschaften. Präsentation für Vortragszwecke. Stand: Februar 2005.

(5) Vgl. § 53 Abs. 1 HGrG (Haushaltsgrundsätzegesetz vom 19. August 1969 (BGBl. I S. 1273), zuletzt geändert durch Artikel 123 der Verordnung vom 31. Oktober 2006 (BGBl. I S. 2407) i.V.m. Fragenkatalog des IDW PW 720 vom 6. Oktober 2006.

(6) Vgl. Bräunig, Dietmar u. Eichhorn, Peter: Konzeption und Kriterien der überörtlichen Prüfung kommunaler Körperschaften in Hessen; Die Wirtschaftsprüfung, Hefte 1/1995 und 2/1995.

(7) Dennoch können sich gerade beim externen Vergleich eine Gegenüberstellung von großen und kleinen Einrichtungen oder ein Vergleich ländlicher und städtischer Einrichtungen wertvolle Erkenntnisse im Hinblick auf Wirtschaftlichkeit ergeben. 